\title{
Algebraic Decay of Velocity Fluctuations in a Confined Fluid
}

\author{
M. H. J. Hagen, ${ }^{1}$ I. Pagonabarraga, ${ }^{1}$ C. P. Lowe, ${ }^{1,2}$ and D. Frenkel ${ }^{1}$ \\ ${ }^{1}$ FOM - Institute for Atomic and Molecular Physics, Kruislaan 407, 1098 SJ Amsterdam, The Netherlands \\ ${ }^{2}$ Computational Physics, Delft University of Technology, Lorentzweg 1, 2628 CJ Delft, The Netherlands
}

(Received 17 January 1997)

\begin{abstract}
Computer simulations of a colloidal particle suspended in a fluid confined by rigid walls show that, at long times, the velocity correlation function decays with a negative algebraic tail. The exponent depends on the confining geometry, rather than the spatial dimensionality. We can account for the tail by using a simple mode-coupling theory which exploits the fact that the sound wave generated by a moving particle becomes diffusive. [S0031-9007(97)03163-3]
\end{abstract}

PACS numbers: 82.70.Dd, 05.40.+j, 66.20.+d, 83.20.Jp

In 1872 , as part of his kinetic theory of gases, Boltzmann [1] introduced the "molecular chaos hypothesis"the assumption that successive collisions experienced by a fluid particle are uncorrelated. For a quantitative description of molecular motion in fluids it is convenient to use the velocity correlation function (v.c.f.). The v.c.f., which we denote by $C_{\alpha}(t)$, is the average of the initial velocity of a particle multiplied with its velocity at a later time $t, C_{\alpha}(t)=\left\langle v_{\alpha}(0) v_{\alpha}(t)\right\rangle$. The molecular chaos hypothesis implies that the velocity correlation function decays exponentially.

It therefore came as a surprise when, in 1970, Alder and Wainwright [2] reported that it did not. They found that in a hard sphere fluid the velocity correlation function decays algebraically with a power that depended on the dimensionality $d$ of the system. They explained their observation of this "long-time tail" in terms of hydrodynamics. This predicts that the momentum of a particle decays by two mechanisms. First, emission of a sound wave: a fraction of the initial momentum is carried away rapidly by a propagating sound mode. This mode does not contribute to the long-time tail. The remaining fraction of the momentum is transported away diffusively. The diffusive transport of momentum is responsible for an algebraic long-time tail of the form $C_{\alpha}(t) \sim t^{-d / 2}$. For a colloidal particle of typical size (one micron), suspended in water, sound propagation only influences the shorttime decay of the v.c.f. (times less than a nanosecond). The time scale for the long-time tail is controlled by the kinematic viscosity of the fluid and is on the order of microseconds. Where we subsequently refer to "long times," we mean this hydrodynamic long time. The time it takes a colloidal particle to significantly move is still longer $\left(\sim 10^{-3} \mathrm{~s}\right)$.

In this Letter we consider the dynamics of a colloidal particle suspended in a fluid confined by rigid walls. Because of the friction exerted by the walls, one would expect that the long-time hydrodynamic tail will be lost and the v.c.f. will decay exponentially. This was indeed the conclusion reached by Bocquet and Barrat [3], in their theoretical analysis of a particle suspended in a fluid contained between two plates. Here we show that, to our surprise, on a hydrodynamic time scale a long-time tail is recovered. What is more, this long-time tail has a different physical origin — and sign — than the tail found in an unbounded fluid.

First, we describe the results of the computer simulations. We used a lattice Boltzmann model to describe the fluid. The state of the fluid is specified by the average number of particles, $n(\mathbf{c}, \mathbf{r}, t)$, with velocity $\mathbf{c}$, at each lattice site $\mathbf{r}$. The time evolution of the distribution functions is described by the discretized analog of the Boltzmann equation [4]. This involves propagation and collision. Collisions are specified such that the time evolution of the hydrodynamic fields satisfies the linearized Navier-Stokes equations for an isothermal, compressible fluid [4]. The boundary conditions at the (stationary) confining walls could be varied between stick and slip. In all cases, stick boundary conditions were imposed on the interface between the colloidal particle and fluid. The equations of motion were integrated using the self-consistent method described in Ref. [5]. We calculated the velocity correlation function by giving an initial velocity, $v_{\alpha}(0)$, to a colloidal particle in an otherwise quiescent lattice Boltzmann fluid. There are no spontaneous fluctuations in the system. However, correlating $v_{\alpha}(0)$ with the subsequent velocity $v_{\alpha}(t)$ is, according to Onsager's regression hypothesis, equivalent to calculating the v.c.f. in a "real" fluctuating system. Our units are such that the mass of the lattice-gas particle, the lattice spacing, and the time step are all unity, the kinematic viscosity $\nu$ of the fluid was equal to $\frac{1}{6}$, the density $\rho$ was 24 , and the speed of sound $c$ was $1 / \sqrt{2}$. The mass of the object was set to correspond to neutral buoyancy. In all cases the v.c.f. was only calculated for times less than the time it takes for a sound wave to cross the system, so there are no finite-size effects to consider.

The first geometry we considered was a cylindrical tube. In Fig. 1 we show the v.c.f., calculated in a direction along the axis of the tube, for a colloidal particle located at the center. We have expressed time in dimensionless units, $\tau=t \nu / r^{2}$, where $r$ is the particle 


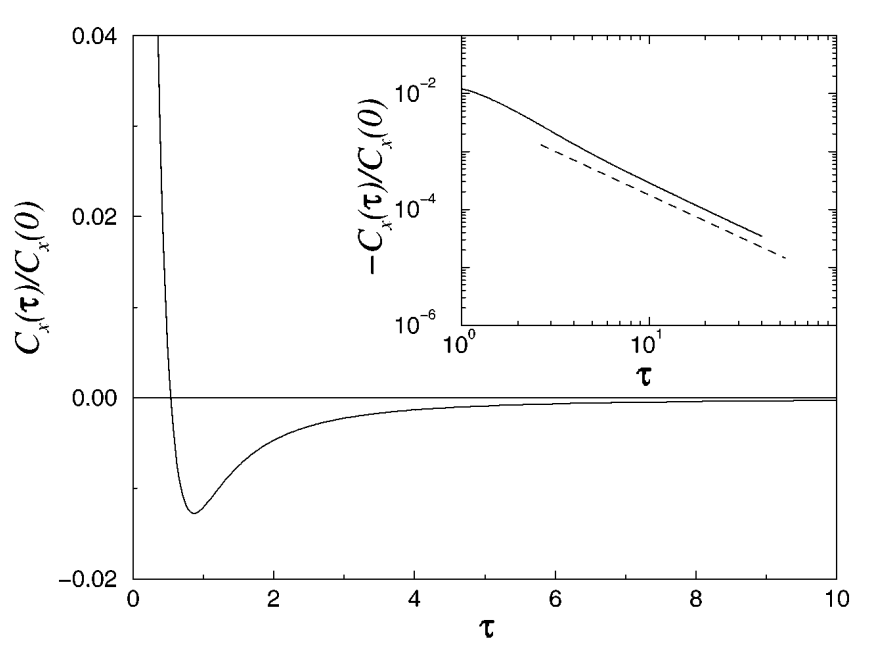

FIG. 1. Normalized velocity correlation function $C_{x}(\tau) / C_{x}(0)$ of a colloidal particle with radius $r=2.5$ in a cylindrical tube with radius $R=4.5$ (drawn line). The dashed line denotes a line with $C_{x}(\tau) \sim-1 / \tau^{3 / 2}$. The reduced time $\tau$ is defined by $\tau=t \nu / r^{2}$.

radius. For an incompressible fluid this is the only relevant parameter. However, if compressibility effects are important, one should also consider the ratio $\mathrm{cr} / \nu$, which is the time it takes transverse momentum to diffuse a particle radius divided by the time it takes sound to travel the same distance. In the simulation this ratio is 10.6 (for a colloidal particle in water the ratio is of order 1000, whereas for olive oil it is 10). The most noticeable feature of the v.c.f. is that it becomes negative. In the absence of the tube, the v.c.f. is positive at all times. We do not see an exponential decayas the inset shows the asymptotic decay is algebraic with the form $C_{x}(t) \sim-1 / t^{3 / 2}$. We observed no such effect for the component of the v.c.f. perpendicular to the tube axis. In this case the decay was exponential. To examine the effect of dimensionality, we studied a two-dimensional system, consisting of a disk in a fluid confined between two walls. Again the same qualitative behavior was found; the v.c.f. becomes negative and decays algebraically. The asymptotic decay has the form $C_{x}(t) \sim-1 / t^{3 / 2}$. This is the same as the result we obtained for the tube in three dimensions. To examine the effect of the confining geometry, we studied a threedimensional fluid contained between two plates. In this case we still observed a negative long-time tail for the v.c.f., parallel to the plates, but now with an exponent -2 instead of $-3 / 2$. This suggests that the exponent of the long-time tail depends on the number of dimensions which are not geometrically confined, $d^{*}$, rather than on the actual spatial dimension. In order to shed more light on the mechanism underlying this behavior, we repeated the calculation for the two walls, but changed the boundary conditions at the walls. The algebraic decay persists as long as the walls exert friction. Only for pure slip boundary conditions do we recover the usual behavior of the v.c.f. of an effectively one dimensional system, namely, exponential decay parallel to the tube axis. For a three dimensional fluid between parallel plates the v.c.f. decays as $1 / t$ in the same limit. From this we concluded that the existence of velocity gradients close to the walls, induced by the boundary conditions, are essential for the negative long-time tail.

In order to clarify the origin of such algebraic tails, we considered the long-time decay of a velocity perturbation in an initially quiescent fluid. For simplicity, we discuss the two-dimensional case with an initial perturbation $\mathbf{v}(\mathbf{r}, 0)=\left(v_{0} \delta(\mathbf{r}), 0\right)$. The evolution of this disturbance is determined by the usual hydrodynamic equations,

$$
\begin{gathered}
\frac{\partial \rho}{\partial t}+\rho_{0} \nabla \cdot \mathbf{v}=0 \\
\frac{\partial \mathbf{v}}{\partial t}+\alpha \nabla \rho-\nu \nabla^{2} \mathbf{v}-\xi \nabla \nabla \cdot \mathbf{v}=0
\end{gathered}
$$

where $\mathbf{v}(\mathbf{r}, t)$ and $\rho(\mathbf{r}, t)$ are the disturbance velocity and density field, respectively, $\rho_{0}$ is the equilibrium density, $\nu$ the shear viscosity, $\xi$ the bulk viscosity, and $\alpha=c^{2} / \rho_{0}$, with $c$ the velocity of sound [for a three dimensional fluid, $\xi$ in Eq. (2) is replaced by $\xi+\nu / 3]$. Although stick boundary conditions should be supplemented to Eqs. (1) and (2), we will introduce them in an effective way. In fact, we assume that at long times the transverse component of the velocity field has almost relaxed $\left(\partial_{x} v_{x} \gg \partial_{y} v_{y}\right)$, and we keep the $y$ dependence of $v_{x}$ (note that for slip, this last requirement is no longer necessary). The solution of this problem reads, in Fourier space,

$$
\tilde{\boldsymbol{v}}_{x}\left(k_{x}, k_{y}, \omega\right)=\frac{i \omega v_{0}}{-\omega^{2}+i \omega\left(\Gamma k_{x}^{2}+\nu k_{y}^{2}\right)+c^{2} k_{x}^{2}},
$$

with $\Gamma=\nu+\xi$, the sound wave damping coefficient. Because of the anisotropy induced by the $y$ dependence of the $x$ component of the velocity, this equation shows that purely diffusive modes can be excited in the tube if $4 c^{2} k_{x}^{2}<\left(\Gamma k_{x}^{2}+\nu k_{y}^{2}\right)^{2}$, implying that, for a fixed value of $k_{y}$, there will always be a $k_{x}^{*}$ such that when $k_{x}<k_{x}^{*}$ only diffusive modes show up. In general, due to the fact that there exists a minimum $k_{y}$ (because of the finite width of the tube), there will always exist a fraction of the modes that are overdamped and will behave diffusively. In the hydrodynamic regime $\left(k_{x} \rightarrow 0\right)$ the modes in the system are

$$
\begin{aligned}
& \omega_{1} \sim i \nu k_{y}^{2}+i\left(\Gamma-\frac{c^{2}}{\nu k_{y}^{2}}\right) k_{x}^{2}+\mathcal{O}\left(k_{x}^{4}\right), \\
& \omega_{2} \sim i \frac{c^{2}}{\nu k_{y}^{2}} k_{x}^{2}+\mathcal{O}\left(k_{x}^{4}\right) .
\end{aligned}
$$

The mode $\omega_{1}$ induces a perturbation which decays exponentially with time because of the minimum value of $k_{y}$. However, in the limit $k_{x} \rightarrow 0$ the $\omega_{2}$ mode gives rise to a 
diffusive perturbation. The prefactor multiplying $k_{x}^{2}$ can be interpreted as an effective diffusion coefficient which characterizes the diffusion of density perturbations, which scales as

$$
D^{*} \sim \frac{c^{2} R^{2}}{\nu},
$$

where $R$ is half the width of the channel. The possibility of exciting such overdamped sound modes has been discussed in Ref. [6], where the hydrodynamics of a thin fluid layer in contact with a solid substrate is modeled as a 2D fluid with an extra dissipation force accounting for the liquid-solid interaction. Recently, it has been argued that sound waves in fluid membranes may also be overdamped [7]. This suggests that the dynamics of particles embedded in such membranes will exhibit the same features reported in this Letter.

The power law characterizing the decay of this second mode can be obtained by transforming back the velocity to real space and time from Eq. (3). An asymptotic analysis of this time decay leads to

$$
v_{x}(t) \sim-\frac{v_{0}}{\sqrt{\nu} t^{3 / 2}}+\mathcal{O}\left(t^{-5 / 2}\right) .
$$

This is in agreement with the simulations of the twodimensional system. An analogous analysis for the threedimensional fluid between two plates shows that

$$
v_{x}(t) \sim-\frac{v_{0}}{t^{2}}+\mathcal{O}\left(t^{-3}\right),
$$

again in agreement with the simulation [8].

The derivation considers a fluid element, but the result should not depend on the specific way the momentum has been introduced, it only depends on the amount of momentum which is inserted. By changing the viscosity of the fluid, we have also verified that the tails scale with the viscosity as predicted by Eqs. (7) and (8).

In order to investigate the diffusive decay of sound, predicted by Eq. (6), we performed a simulation in which we actually measured this effective diffusion constant. To do this we increased the density of the fluid at a point in the center of a two dimensional slit, and computed the second moment of the evolving spatial density distribution. The diffusion coefficient was obtained by differentiation: $D^{*}=\frac{1}{2} \frac{d}{d t}\left\langle r^{2}(t)\right\rangle$. The diffusion coefficient is plotted in Fig. 2, as a function of the kinematic viscosity. Changing the channel width and the viscosity, we find

$$
D^{*}=\frac{c^{2}\left(R^{2}-1 / 4\right)}{3 \nu}+\frac{1}{2},
$$

which is in agreement with Eq. (6) (except for the factors of $-1 / 4$ [4] and 1/2 [9] which are, in fact, lattice artifacts).

This analysis allows us to give a more intuitive picture for the appearance of the negative algebraic decay. The initial motion of the particle in the tube sets up a density dipole in the fluid. If we consider that only overdamped

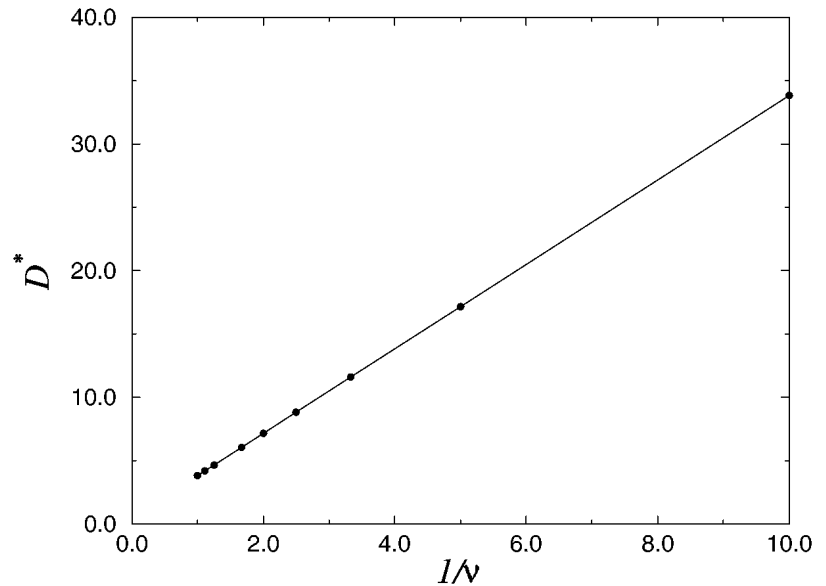

FIG. 2. The effective diffusion coefficient of density perturbations $D^{*}$ as a function of $1 / \nu$, where $\nu$ is the dimensionless kinematic viscosity. Results were obtained in a two-dimensional slit of half width $R=4.5$. The points denote the simulation results, and the line is a guide to the eye.

modes are present in the system, this density dipole will decay diffusively,

$$
\rho(x, t)=\frac{x \exp \left(-x^{2} / 4 D^{*} t\right)}{\sqrt{4 \pi\left(D^{*} t\right)^{3}}},
$$

where $x$ is the distance from the particle along the tube and the density profile in the transverse direction is essentially flat. This leads to a mass flux around the origin which is proportional to the gradient in density $j(0, t) \sim$ $-\partial_{x} \rho(0, t)$. The particle (or fluid element) which caused the initial dipole will now (at long times) be enslaved to this mass flux, and the flow related to the flux will be the velocity of the particle $\left[v(t) \sim-1 / t^{3 / 2}\right]$. For the particle diffusing between two plates (in three dimensions), the diffusion of the density will be two dimensional, and, arguing along the same lines, we find $v(t) \sim-1 / t^{2}$. This shows that the long-time tail is driven by a pressure relaxation mechanism. From here we argue that this result is general and that

$$
C_{x}(t) \sim-\frac{1}{t^{1+d^{*} / 2}} .
$$

The requirement for finding this behavior is that the geometry overdamps sound modes. The result in Eq. (11) differs from the velocity correlation function found by Bocquet and Barrat [3], where a simple mode-coupling approach was used [10], which neglected the coupling of velocity with sound at long times. We also note that the form of v.c.f. we observe is the same as that found in a purely diffusive system-the Lorentz gas [11]. The physical origins of the two effects are, however, quite different.

By integrating the v.c.f. we can calculate the diffusion coefficient for a particle in a tube to see whether this long-time tail contributes to the diffusion coefficient. The results are shown in Fig. 3, normalized by the diffusion 


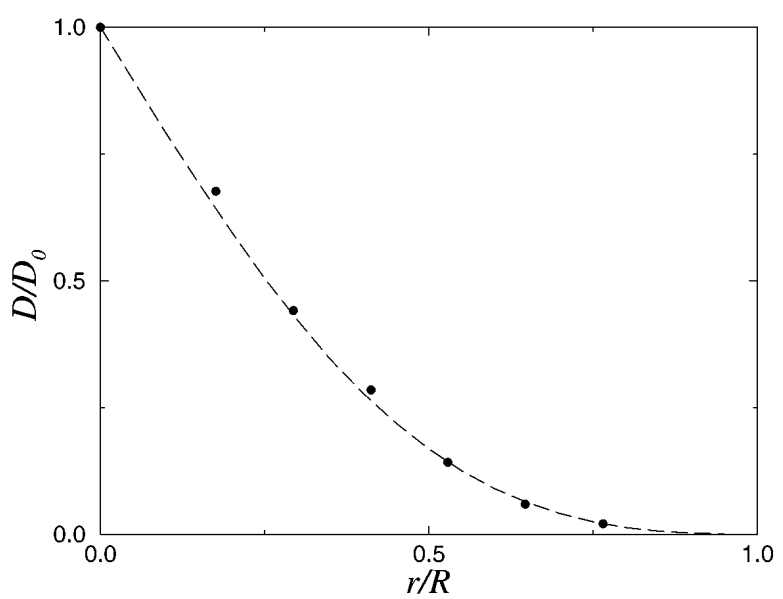

FIG. 3. Normalized diffusion coefficient of a particle with radius $r$ in the center of a cylindrical tube with radius $R$. The points denote simulation results, and the line corresponds to the centerline approximation (Ref. [12]).

coefficient in the absence of the tube $D_{0}$. Also plotted is the centerline approximation [12], calculated assuming a purely incompressible fluid (neglecting sound effects). The simulations lead to the same diffusion coefficient as predicted by the theory for incompressible fluids. In fact, this must be the case because the long-time tail originates from the compressible modes. These modes do not affect the integral of the v.c.f. - the contribution from Eq. (3) vanishes in the limit $\omega \rightarrow 0$. The diffusion coefficient is determined by the decay of transverse velocity perturbations $[3,13]$ which, in a confined system, is exponential. So, while compressibility effects dominate the long-time dynamics, they still do not contribute to the diffusion coefficient.

Experimentally, one can expect to observe the longtime tail most easily if all the modes are overdamped, that is, when $k_{y}^{2}>\frac{2 c}{\nu} k_{x}$. If we consider a colloidal particle of radius $r$ in a cylindrical tube of radius $R$ and length $L$, this condition is satisfied if

$$
\left(\frac{r}{R}\right)^{2}>\frac{4}{\pi} \frac{c r}{\nu} \frac{r}{L}
$$

If we consider $\frac{r}{L} \sim 10^{-4}, \frac{r}{R} \sim \frac{1}{3}$, for water $(c \approx$ $1.5 \times 10^{5} \mathrm{~cm} / \mathrm{s}, \nu \approx 10^{-2} \mathrm{~cm}^{2} / \mathrm{s}$ ) we obtain the restriction that $r<5 \times 10^{-5} \mathrm{~cm}$, which is reasonable for colloidal particles. For a more viscous fluid, such as olive oil, the estimate is $r<5 \times 10^{-3} \mathrm{~cm}$. Here we considered a single particle in a tube, but, if particle-particle interactions within the pore are small compared with particle-wall interactions, the analysis also holds for a suspension of colloidal particles. A rough estimate of the ap- propriate volume fraction, $\phi$, for which such condition is satisfied is given in Ref. [14]: $\phi<\frac{2}{3} \frac{r}{R}$, with $\phi$ the solute volume fraction in the pore.

In summary, our simulations show that the v.c.f. of a colloidal particle in a fluid confined by rigid walls can display a negative long-time tail. The mode-coupling analysis suggests that the mechanism driving the longtime tail is the diffusive decay of density perturbations that would normally decay by sound propagation. The simulations support this. At long-times sound propagation plays no part for a particle in an unbounded fluid-in a confined fluid it is the dominant effect.

The work of the FOM Institute is part of the scientific program of FOM and is supported by the Nederlandse Organisatie voor Wetenschappelijk Onderzoek (NWO). Computer time on the CRAY-C98/4256 at SARA was made available by the Stichting Nationale Computer Faciliteiten (Foundation for National Computing Facilities). I.P. acknowledges E.U. for its financial support [Contract No. ERBFMBICT-950433], and the FOM-Institute for its hospitality. We gratefully thank Patrick Warren for his useful comments and Lyderic Bocquet, Matthieu Ernst, and Bela Mulder for critical readings of the manuscript.

[1] L. Boltzmann, Wien. Ber. 66, 275 (1872).

[2] B. J. Alder and T.E. Wainwright, Phys. Rev. A 1, 18 (1970).

[3] L. Bocquet and J.-L. Barrat, J. Phys. Condens. Matter 8, 9297 (1996).

[4] A. J. C. Ladd, J. Fluid Mech. 271, 285 (1994).

[5] C. P. Lowe, D. Frenkel, and A. J. Masters, J. Chem. Phys. 103, 1582 (1995).

[6] S. Ramaswamy and G. F. Mazenko, Phys. Rev. A 26, 1735 (1982).

[7] W. Cai and T. C. Lubenski, Phys. Rev. Lett. 73, 1186 (1994).

[8] M.H.J. Hagen, I. Pagonabarraga, C.P. Lowe, and D. Frenkel (to be published).

[9] T. Naitoh, M.H. Ernst, M. A. van der Hoef, and D. Frenkel, Phys. Rev. A 44, 2484 (1991).

[10] M. H. Ernst, E. H. Hauge, and J. M. J. van Leeuwen, Phys. Rev. Lett. 25, 1254 (1970).

[11] M. H. Ernst and A. Weyland, Phys. Lett. 34A, 39 (1971).

[12] P. M. Bungay and H. Brenner, Int. J. Multiph. Flow 1, 25 (1973).

[13] L. Bocquet and J.-L. Barrat, Europhys. Lett. 31, 455 (1995).

[14] H. Happel and H. Brenner, Low Reynolds Number Hydrodynamics (Prentice-Hall, Englewood Cliffs, NJ, 1965). 